Doi: $10.32481 / \mathrm{djph} .2021 .12 .012$

\title{
Impact of the Access to Genetic Counselor Services Act
}

\author{
Carol Nowlen, $\mathrm{MS}^{1}$ and Kendra Flores, MS, CGC ${ }^{2}$ \\ 1. Genetic Counselor, ChristianaCare \\ 2. Senior Licensed Genetic Counselor, ChristianaCare
}

\begin{abstract}
The goal of this Opinion Editorial is to raise public awareness regarding legislation introduced to Congress, known as the Access to Genetic Counselor Services Act, or HR 2144/S1450. Currently, Medicare policy denies beneficiaries direct access to genetic counselors. This bill aims to rectify this though CMS recognition of genetic counselors. This is of particular importance to Delawareans who rely on genetic counseling service in the sub-specialties of cancer, preconception/prenatal, pediatrics, cardiology, and neurology. In this paper, we aim to underscore the importance of this legislation by outlining how genetic counselors impact patient care.
\end{abstract}

The Access to Genetic Counselor Services Act, known as either S.1450 or HR.2144, is a bill that has been introduced to Congress with the intent to increase access to genetic counselors. Currently, Medicare and Medicaid policy denies beneficiaries access to genetic counselors. S.1450/HR.2144 aims to rectify this by having the Centers for Medicare and Medicaid Services (CMS) recognize genetic counselors as standalone healthcare providers. Due to the rapid expansion of genetic knowledge across medicine, genetic expertise is essential to a growing number of specialties. This bill would help ensure that Delawareans will have reliable access to cost-saving allied health professionals with niche training in this evolving field.

Genetic counselors are highly trained professionals that work collaboratively as members of the medical team. Genetic counselors in the United States obtain a master's degree from one of 52 programs accredited by the Accreditation Council for Genetic Counseling. Graduation requirements include rigorous coursework, field placement, and completion of a graduate-level thesis. In addition to genetics expertise, the curriculum emphasizes interpersonal, psychosocial, and counseling skills. Graduates that pass a board examination administered by the American Board of Genetic Counseling are designated a title of certified genetic counselor. Currently, 28 states grant licensure for the practice of genetic counseling with all but two states working towards establishing licensure in the future.

In an appointment with a genetic counselor, patients can be expected to be asked questions regarding family members and their health history, as well as the patient's personal health history. Important things to note when considering personal and family histories are congenital conditions, sudden or unexplained deaths, conditions that occur much earlier in individuals than they do in the general population (such as cancer, aneurysms, strokes, vision and hearing loss, etc.), and any known genetic diagnoses. It is also important to note any family members that have already had genetic testing, and what kind. Based on personal and family history, the genetic counselor will then review the concept of genetics, the likelihood of a genetic diagnosis for the family, and testing that may be available. The conversation would then be centered around benefits and limitations of testing while facilitating decision making by helping patients evaluate how the testing aligns with their values and concerns. 
Genetic counselors work within several subspecialty clinics in Delaware, including:

- Cancer: genetic counselors are members of the oncology team that evaluate the risk that an individual has a genetic condition that can cause an increased risk for cancer. Examples include Hereditary Breast and Ovarian Cancer syndrome (BRCA1/BRCA2) and Lynch syndrome.

- Prenatal/Preconception: genetic counselors work with reproductive endocrinologists, obstetricians and gynecologists, and maternal fetal medicine specialists to evaluate the risk that a couple would have a pregnancy affected with a genetic condition.

- Pediatrics: genetic counselors work with a geneticist to care for children that may have genetic conditions. Common referral reasons include birth defects, diagnoses of autism spectrum disorder, or abnormal newborn screening tests.

- Neurology: genetic counselors that work in neurology can work in a team with a geneticist or a neurologist to see patients with neurological conditions, like seizures or unexplained neuropathy.

- Cardiovascular: genetic counselors work, often with cardiologists, to evaluate various cardiac concerns, including aortic dissections, arrhythmias, cardiomyopathies or family histories of sudden unexplained deaths.

- Transthyretin Amyloidosis: a specialized clinic in Delaware that serves patients with a prevalent but underdiagnosed hereditary form of amyloidosis. This disease results in a misfolded protein which can cause multisystemic dysfunction. Notably, the diagnosis of this condition can allow for gene-specific tailored interventions.

- Huntington Disease: a multi-disciplinary clinic which includes a genetic counselor, clinical psychologist and neurologists who evaluate the readiness for diagnosis in individuals with symptoms or family histories of Huntington disease.

- General Genetics: genetic counselors work with geneticists to evaluate individuals that may have adult-onset genetic conditions that do not fit neatly into a different subspecialty. Examples include mitochondrial conditions and connective tissue disorders.

It is an important point of discussion that CMS recognizes the act of genetic counseling. The procedural (CPT) code 96040 allows providers to bill for the act of analyzing a family history, risk-assessment, and counseling. While CMS will provide payment for this code when billed by a physician, nurse practitioner or physician assistant, reimbursement is not granted when performed by a genetic counselor. This denial is attributed to the fact that CMS does not recognize genetic counselors as medical providers.

While policies vary widely by location, several third-party insurers reimburse genetic counselors for their service since the inception of the procedural code for genetic counseling in 2007. In the greater Delaware area, most private insurance companies recognize the value genetic counselors bring to their subscribers and will reimburse a genetic counselor for this CPT code. However, many private insurance companies look to CMS to inform their coverage policies. As a result, some genetic counselors are not reimbursed by these companies for the service they provide. In 
cases where insurance will not pay, the facility the genetic counselor is employed through may choose to bill instead. These facility fees provide significantly less reimbursement than the CPT code and can only be utilized when the appointment occurred within the billing facility. Ergo, even though genetic counseling is perfectly suited for remote healthcare (telemedicine) because the sessions are discussion-based, it is often a non-covered service modality.

Additionally, it has been frequently demonstrated that genetic counselors save healthcare dollars. Genetic counselors are uniquely qualified to ensure the correct test is performed, the correct family member is tested, and results are correctly interpreted. One 2014 study tracked the costsavings provided by having genetic counselors perform a pre-analytical assessment of the appropriateness of testing, which established that the "test review process resulted in an average reduction in charges to the referring institutions of $\$ 48,000.00$ per month." ${ }^{1}$ Further, S.1450/HR.2144 proposes reimbursement of genetic counselors at $85 \%$ the physician-fee schedule. Consequently, recognition of genetic counselors would save $15 \%$ of the federal dollars already being paid for physicians to provide genetic counseling.

In the age of personalized medicine, access to a specialty-trained genetic expert is invaluable. Current federal policy fails to recognize genetic counselors and the role they play in making genetic information accessible and understandable to the public. We urge you to consider contacting your congressional representative in support of S.1450/HR.2144 or visiting https://www.nsgc.org/Policy-Research-and-Publications/Federal-Advocacy to learn more.

Carol Nowlen, MS, CGC can be contacted at Carol.Nowlen@ChristianaCare.org

\section{References}

1. Miller, C. E., Krautscheid, P., Baldwin, E. E., Tvrdik, T., Openshaw, A. S., Hart, K., \& Lagrave, D. (2014, May). Genetic counselor review of genetic test orders in a reference laboratory reduces unnecessary testing. American Journal of Medical Genetics. Part A, 164(5), 1094-1101. PubMed https://doi.org/10.1002/ajmg.a.36453

Copyright (c) 2021 Delaware Academy of Medicine / Delaware Public Health Association.

This is an Open Access article distributed under the terms of the Creative Commons Attribution Non-Commercial License (https://creativecommons.org/licenses/by-nc-nd/4.0/) which permits unrestricted non-commercial use, distribution, and reproduction in any medium, provided the original work is properly cited. 